\title{
Herrmann-Beller Phosphapalladacycle-Catalyzed Addition of Alkynes to Norbornadienes
}

\author{
Alphonse Tenaglia*, Laurent Giordano and Gérard Buono \\ [*] Dr. A. Tenaglia, Dr. L. Giordano, Prof. Dr. G. Buono \\ Laboratoire de Synthèse Asymétrique, UMR 6180 CNRS. Université d'Aix-Marseille P. \\ Cézanne, Faculté des Sciences et Techniques de St Jérôme, EGIM, Case A62, Avenue \\ Escadrille Normandie Niemen, 13397 Marseille Cedex 20, France. \\ Fax: +33-491-282742 \\ Email: alphonse.tenaglia@univ.u-3mrs.fr
}

\section{- General:}

Reactions were carried out under an argon atmosphere. Commercial reagents were used without further purification. Ether was distilled prior to use from sodium/benzophenone. Petroleum ether (PE) and 1,2-dichloroethane (DCE) were distilled prior to use from calcium hydride. Alkynes not commercially available were prepared according literature procedures. ${ }^{1}$ Methyl-prop-2-ynylcarbonate $3 \mathbf{k},{ }^{2}$ 4,4Dimethyl-hept-6yn-2-one $\mathbf{3} \mathbf{n}^{3}$ and prop-2-ynylsulfanylbenzene $\mathbf{3} \mathbf{p}^{4}$ were prepared using methods as previously described. Analytical thin layer chromatography was performed on silica gel $60 \mathrm{~F}_{254}$ plates $(0.25 \mathrm{~mm})$. Flash chromatography was performed on silica gel 60 (230-400 mesh). Melting points were taken on a capillary apparatus and are uncorrected. IR spectra were recorded as liquid films or $\mathrm{KBr}$ plates. ${ }^{1} \mathrm{H}$ and ${ }^{13} \mathrm{C} \mathrm{NMR}$ were referenced to $\mathrm{CDCl}_{3}$ at $\delta 7.26$ and 77.0 respectively. The different types of carbon in the structures have been identified by DEPT techniques.

- Palladium-Catalyzed Alkynylation of Bicyclo[2.2.1]hepta-2,5-diene. General Procedure.

In a $10 \mathrm{~mL}$ flame-dried Schlenk tube, alkyne 3 (1 mmol) and norbornadiene 2 (184 mg, $2 \mathrm{mmol}$ ) in $6 \mathrm{~mL}$ of 1,2-dichloroethane (DCE) were added to a solution of palladacycle 1 (23.5 mg, $0.025 \mathrm{mmol}$ ) in $2 \mathrm{~mL}$ of DCE under argon. The reaction mixture was stirred under conditions reported in Table 1. The reaction mixture was evaporated in vacuo, and the residue was purified by chromatography on silica gel to give the enyne 4 . 
exo-5-(2-phenylethynyl)bicyclo[2.2.1]hept-2-ene (4a)

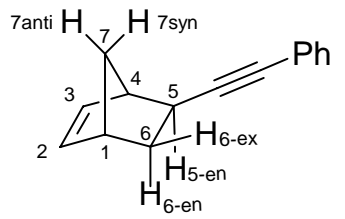

$4 \mathbf{a}$

Chemical yield : 90\%. Colorless oil. Rf (PE) 0.84; IR (neat) v 3058, 2219, 1604, $1578 \mathrm{~cm}^{-1} ;{ }^{1} \mathrm{H}$ NMR $\left(\mathrm{CDCl}_{3}, 500 \mathrm{MHz}\right) \delta 1.48$ (br d, $J=8.5 \mathrm{~Hz}, 1 \mathrm{H}, \mathrm{H}_{7 \text { anti }}$ ), 1.56 (ddd, $J$ $\left.=2.3,8.7,11.5 \mathrm{~Hz}, 1 \mathrm{H}, \mathrm{H}_{6-\mathrm{en}}\right), 1.68$ (d, $\left.J=8.5 \mathrm{~Hz}, 1 \mathrm{H}, \mathrm{H}_{7 \mathrm{syn}}\right), 1.78$ (dt, $J=3.8,11.5 \mathrm{~Hz}$, $1 \mathrm{H}, \mathrm{H}_{6-\mathrm{ex}}$ ), 2.34 (ddd, $\left.J=0.9,4.0,8.7 \mathrm{~Hz}, 1 \mathrm{H}, \mathrm{H}_{5-\mathrm{en}}\right), 2.95$ (m, 1H, $\left.\mathrm{H}_{1}\right), 3.01$ (m, $1 \mathrm{H}$, $\mathrm{H}_{4}$ ), 6.07 (dd, $J=2.9,5.5 \mathrm{~Hz}, 1 \mathrm{H}, \mathrm{H}_{3}$ ), 6.11 (dd, $J=2.9,5.5 \mathrm{~Hz}, 1 \mathrm{H}, \mathrm{H}_{2}$ ), 7.25-7.29 (m, 3H), 7.39 (m, 2H); ${ }^{13} \mathrm{C} \mathrm{NMR}\left(\mathrm{CDCl}_{3}, 50 \mathrm{MHz}\right) \delta 29.5$ (d), 34.8 (t), 41.9 (d), 46.8 (t), 49.3 (d), 80.3 (s), 95.0 (s), 124.1 (s), 127.4 (d), 128.1 (d), 131.5 (d), 135.3 (d), 137.4 (d). Anal. Calcd for $\mathrm{C}_{15} \mathrm{H}_{14}$ : C, 92.74; H, 7.26. Found: C, 92.83; H, 7.14.

exo-6-Deuterio-exo-5-phenylethynyl-bicyclo[2.2.1]hept-2-ene D-(4a)

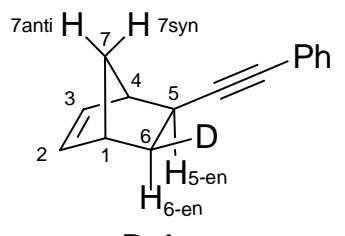

D-4a

D-(4a) was prepared in a similar manner as for 4a (see above general procedure) but using norbornadiene 2 as solvent. The exo position of deuterium at C-6 was based on the disappearance of the signal of $\mathrm{H}_{6 \text {-ex }}$ as a dt at $\delta 1.78$ and the resolution of signals for $\mathrm{H}_{5-\text { en }}$ and $\mathrm{H}_{6-\text { en }}$ as a d $(J=8.7 \mathrm{~Hz})$ in comparison with data for 4 a.

Chemical yield : 92\%. Colorless oil. ${ }^{1} \mathrm{H}$ NMR $\left(\mathrm{CDCl}_{3}, 500 \mathrm{MHz}\right) \delta 1.48$ (d, $J=8.5 \mathrm{~Hz}$, 1H, $\mathrm{H}_{7 \text { anti }}$ ), 1.54 (d, $\left.J=8.7 \mathrm{~Hz}, 1 \mathrm{H}, \mathrm{H}_{6 \text {-en }}\right), 1.68$ (d, $J=8.5 \mathrm{~Hz}, 1 \mathrm{H}, \mathrm{H}_{7 \mathrm{syn}}$ ), 2.34 (d, $J=$ $8.7 \mathrm{~Hz}, 1 \mathrm{H}, \mathrm{H}_{5-\mathrm{en}}$ ), 2.95 (m, 1H, $\mathrm{H}_{1}$ ), 3.01 (m, 1H, $\mathrm{H}_{4}$ ), 6.07 (dd, $J=2.9,5.5 \mathrm{~Hz}, 1 \mathrm{H}$, $\mathrm{H}_{3}$ ), 6.11 (dd, $\left.J=2.9,5.5 \mathrm{~Hz}, 1 \mathrm{H}, \mathrm{H}_{2}\right), 7.25-7.29$ (m, 3H), $7.39(\mathrm{~m}, 2 \mathrm{H}) ;{ }^{13} \mathrm{C} \mathrm{NMR}$ $\left(\mathrm{CDCl}_{3}, 50 \mathrm{MHz}\right) \delta 29.5$ (d), [34.0, 34.4 and 34.8 (C-6)], 41.8 (d), 46.8 (t), 49.3 (d), 80.2 (s), 95.0 (s), 124.1 (s), 127.4 (d), 128.1 (d), 131.5 (d), 135.3 (d), 137.4 (d). 


\section{5-(hex-1-ynyl)bicyclo[2.2.1]hept-2-ene (4b)}

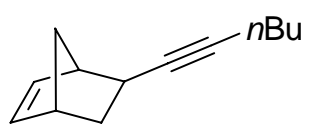

4b

Chemical yield : 72\%. Colorless oil. $R f$ (PE) 0.89; IR (neat) v 3066, 2218, $1554 \mathrm{~cm}^{-1}$; ${ }^{1} \mathrm{H} \mathrm{NMR}\left(\mathrm{CDCl}_{3}, 200 \mathrm{MHz}\right) \delta 0.88$ (t, $\left.J=6.8 \mathrm{~Hz}, 3 \mathrm{H}\right), 1.43$ (m, 8H), 2.10 (m, 3H), 2.82 (m, 2H), 6.00 (m, 2H); $\left.{ }^{13} \mathrm{C} \mathrm{NMR} \mathrm{(CDCl} 3,50 \mathrm{MHz}\right) \delta 13.6(\mathrm{q}), 18.8(\mathrm{t}), 21.9$ (t), $28.9(\mathrm{~d})$, 31.3 (t), 34.9 (t), 41.8 (d), 46.6 (t), 49.3 (d), 79.8 (s), 85.0 (s), 135.3 (d), 137.0 (d). Anal. Calcd for $\mathrm{C}_{13} \mathrm{H}_{18}$ : C, 89.59; H, 10.41. Found: C, 89.72; H, 10.28 .

\section{5-(2-cyclohexenylethynyl)bicyclo[2.2.1]hept-2-ene (4c)}

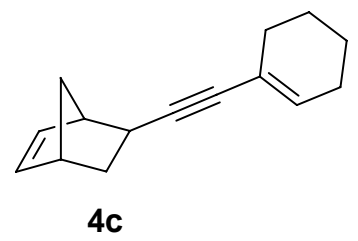

Chemical yield : 87\%. Colorless oil. $R f$ (PE) 0.84; IR (neat) v 3059, 2211, 1682, 1633 $\mathrm{cm}^{-1} ;{ }^{1} \mathrm{H}$ NMR $\left(\mathrm{CDCl}_{3}, 200 \mathrm{MHz}\right) \delta 1.56$ (m, 8H), 2.07 (m, 4H), 2.19 (m, 1H), 2.88 (m, 2H), $6.01(\mathrm{~m}, 3 \mathrm{H}) ;{ }^{13} \mathrm{C} \mathrm{NMR}\left(\mathrm{CDCl}_{3}, 50 \mathrm{MHz}\right) \delta 21.6(\mathrm{t}), 22.4(\mathrm{t}), 25.5(\mathrm{t}), 29.3(\mathrm{~d})$, $29.6(\mathrm{t}), 34.9$ (t), 41.8 (d), 46.7 (t), 49.3 (d), 81.9 (s), 92.0 (s), 121.1 (s), 133.0 (d), 135.3 (d), 137.2 (d). Anal. Calcd for $\mathrm{C}_{15} \mathrm{H}_{18}$ : C, 90.85; H, 9.15. Found: C, 90.72; H, 9.01.

\section{(2-(bicyclo[2.2.1]hept-5-en-2-yl)ethynyl)trimethylsilane (4d)}

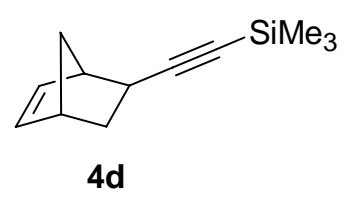

Chemical yield : 93\%. Colorless oil. Rf (PE) 0.94; IR (neat) v 2964, 2172, 1604, $1254 \mathrm{~cm}^{-1}$; ${ }^{1} \mathrm{H}$ NMR $\left(\mathrm{CDCl}_{3}, 200 \mathrm{MHz}\right) \delta 0.12$ (s, 9H), 1.41 (m, 2H), 1.54 (m, 1H), 1.67 (dt, $J=11.3,3.6 \mathrm{~Hz}, 1 \mathrm{H}), 2.12(\mathrm{~m}, 1 \mathrm{H}), 2.88$ (m, 2H), 6.02 (m, $2 \mathrm{H}) ;{ }^{13} \mathrm{C} \mathrm{NMR}\left(\mathrm{CDCl}_{3}\right.$, $50 \mathrm{MHz}) \delta 0.2$ (q), 29.9 (d), 34.9 (t), 41.8 (d), 46.7 (t), 49.4 (d), 83.6 (s), 112.3 (s), 135.3 (d), 137.3 (d). Anal. Calcd for $\mathrm{C}_{12} \mathrm{H}_{18} \mathrm{Si}$ : C, 75.72; H, 9.53. Found: C, 75.58; H, 9.37. 
exo-5-(3-phenoxyprop-1-ynyl)bicyclo[2.2.1]hept-2-ene (4e)

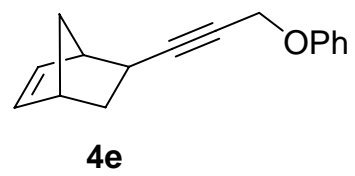

Chemical yield : 80\%. Colorless oil. $R f\left(\mathrm{PE}_{\mathrm{Et}} \mathrm{O}, \mathrm{O}, 95 / 5\right)$ 0.78; IR (neat) $v 3067,2227$, 1603, $1592 \mathrm{~cm}^{-1} ;{ }^{1} \mathrm{H}$ NMR $\left(\mathrm{CDCl}_{3}, 200 \mathrm{MHz}\right) \delta 1.46$ (m, 3H), 1.66 (dt, $J=3.6,11.3$ Hz, 1H), 2.15 (m, 1H), 2.89 (m, 2H), 4.69 (d, $J=2.1 \mathrm{~Hz}, 2 \mathrm{H}) 6.00$ (dd, $J=3.1,4.9 \mathrm{~Hz}$, 1H), 6.06 (dd, $J=3.2,4.8 \mathrm{~Hz}, 1 \mathrm{H}), 6.95$ (m, 3H), 7.29 (m, 2H); ${ }^{13} \mathrm{C} \mathrm{NMR}\left(\mathrm{CDCl}_{3}, 50\right.$ MHz) $\delta 28.9$ (d), 34.4 (t), 41.7 (d), 46.7 (t), 48.9 (d), 56.5 (t), 74.6 (s), 92.7 (s), 114.9 (d), 121.1 (d), 129.2 (d), 135.1 (d), 137.2 (d), 157.8 (s). Anal. Calcd for $\mathrm{C}_{16} \mathrm{H}_{16} \mathrm{O}: \mathrm{C}$, 85.68; H, 7.19. Found: C, 85.56; H, 7.12.

exo-5-(3-(benzyloxy)prop-1-ynyl)bicyclo[2.2.1]hept-2-ene (4f)

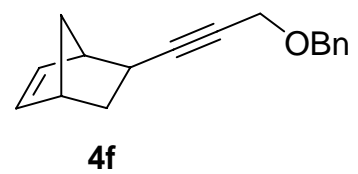

Chemical yield : 95\%. Colorless oil. $R f\left(\mathrm{PE}_{\mathrm{Et}} \mathrm{O}\right.$, 95/5) 0.60; IR (neat) v 3059, 2219, 1704, 1667, $1610 \mathrm{~cm}^{-1} ;{ }^{1} \mathrm{H}$ NMR $\left(\mathrm{CDCl}_{3}, 200 \mathrm{MHz}\right) \delta 1.55$ (m, 3H), 1.65 (dt, $J=4.0$, $11.6 \mathrm{~Hz}, 1 \mathrm{H}), 2.74$ (m, 1H), 2.89 (m, 2H), 4.16 (d, $J=2.0 \mathrm{~Hz}, 2 \mathrm{H}), 4.57$ (s, 2H), 6.02 (m, 2H), 7.33 (m, 5H); ${ }^{13} \mathrm{C} \mathrm{NMR}\left(\mathrm{CDCl}_{3}, 50 \mathrm{MHz}\right) \delta 28.9$ (d), 34.5 (t), 41.7 (d), 46.7 (t), 49.0 (d), 57.7 (t), 71.1 (t), 75.5 (s), 91.8 (s), 127.6 (d), 127.9 (d), 128.2 (d), 135.1 (d), 137.2 (d), 137.6 (s). Anal. Calcd for $\mathrm{C}_{17} \mathrm{H}_{18} \mathrm{O}$ : C, 85.67; H, 7.61. Found: C, 85.53; H, 7.72 .

(3-(bicyclo[2.2.1]hept-5-en-2-yl)prop-2-ynyloxy)(tert-butyl)dimethylsilane (4g)

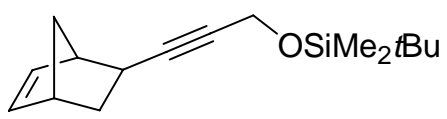

$4 \mathrm{~g}$

Chemical yield : 95\%. Colorless oil. $R f(\mathrm{PE})$ 0.15, $R f\left(\mathrm{PE} / \mathrm{Et}_{2} \mathrm{O}, 98 / 2\right)$ 0.81; IR (neat) v 2964, 2941, 2862, 2227, 1478, 1086, $843 \mathrm{~cm}^{-1}$; ${ }^{1} \mathrm{H}$ NMR $\left(\mathrm{CDCl}_{3}, 200 \mathrm{MHz}\right) \delta 0.1$ (s, 6H), 0.89 (s, 9H), 1.47 (m, 3H), 1.64 (dt, $J=11.4,3.8$ Hz, 1H), 2.12 (ddd, $J=2.1$, 4.2, $8.5 \mathrm{~Hz}, 1 \mathrm{H}), 2.87$ (m, 2H), 4.30 (d, $J=2.1 \mathrm{~Hz}, 2 \mathrm{H}), 6.02(\mathrm{~m}, 2 \mathrm{H}) ;{ }^{13} \mathrm{C} \mathrm{NMR}\left(\mathrm{CDCl}_{3}, 50\right.$ MHz) $\delta$ - 5.0 (q), 18.2 (s), 25.8 (q), 28.9 (t), 34.2 (d), 41.7 (d), 46.7 t), 48.9 (d), 52.0 (t), 
78.4 (s), 89.8 (s), 135.2 (d), 137.2 (d). Anal. Calcd for $\mathrm{C}_{16} \mathrm{H}_{26} \mathrm{OSi}$ : C, 85.68; H, 7.19. Found: C, 85.56; H, 7.32.

\section{3-(bicyclo[2.2.1]hept-5-en-2-yl)prop-2-ynyl acetate (4h)}

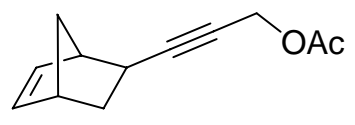

$4 h$

Chemical yield : 67\%. Colorless oil. $R f\left(\mathrm{PE}_{\mathrm{Et}} \mathrm{O}\right.$, 95/5) 0.44; IR (neat) v 3058, 2226, $1751 \mathrm{~cm}^{-1}$; ${ }^{1} \mathrm{H}$ NMR $\left(\mathrm{CDCl}_{3}, 200 \mathrm{MHz}\right) \delta 1.42$ (m, 3H), 1.65 (dt, $\left.J=11.5,3.7 \mathrm{~Hz}, 1 \mathrm{H}\right)$, 2.04 (s, 3H), 2.14 (m, 1H), 2.88 (m, 2H), 4.65 (d, $J=2.1 \mathrm{~Hz}, 2 \mathrm{H}$ ), 5.98 (dd, $J=3.3,5.4$ $\mathrm{Hz}, 1 \mathrm{H}), 6.04$ (dd, $J=3.2,5.5 \mathrm{~Hz}, 1 \mathrm{H}) ;{ }^{13} \mathrm{C} \mathrm{NMR}\left(\mathrm{CDCl}_{3}, 50 \mathrm{MHz}\right) \delta 20.8$ (q), 28.9 (d), 34.4 (t), 41.8 (d), 46.7 (t), 49.0 (d), 52.9 (t), 73.5 (s), 92.1 (s), 135.1 (d), 137.3 (d), 170.3 (s). Anal. Calcd for $\mathrm{C}_{12} \mathrm{H}_{14} \mathrm{O}_{2}$ : C, 75.76; H, 7.42. Found: C, 75.58; H, 7.38.

\section{4-(bicyclo[2.2.1]hept-5-en-2-yl)but-3-yn-2-yl acetate (4i)}

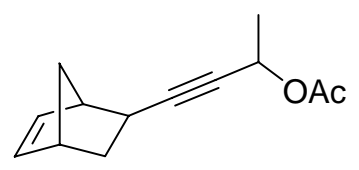

4i

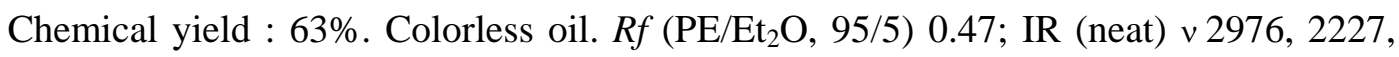
1744, $1237 \mathrm{~cm}^{-1}$; ${ }^{1} \mathrm{H}$ NMR $\left(\mathrm{CDCl}_{3}, 200 \mathrm{MHz}\right) 1.43$ (d, $\left.J=6.6 \mathrm{~Hz}, 3 \mathrm{H}\right), 1.47$ (m, 3H), 1.64 (dt, $J=11.5,4.0 \mathrm{~Hz}, 1 \mathrm{H}), 2.04$ (s, 3H), 2.11 (m, 1H), 2.87 (pseudo dd, $J=1.6,3.3$ Hz, 2H), 5.43 (dq, $J=1.9,6.6 \mathrm{~Hz}, 1 \mathrm{H}$ ), 5.98 (ddd, $J=1.1,3.5,5.8 \mathrm{~Hz}, 1 \mathrm{H}$ ), 6.04 (ddd, $J$ = 1.2, 3.4, $5.7 \mathrm{~Hz}, 1 \mathrm{H}) ;{ }^{13} \mathrm{C} \mathrm{NMR}\left(\mathrm{CDCl}_{3}, 50 \mathrm{MHz}\right) \delta 21.0$ (q), 21.7 (q), 28.6 (d), 34.4 (t), 41.6 (d), 46.5 (t), 48.9 (d), 60.7 (d), 78.1 (s), 89.8 (s), 135.0 (d), 137.1 (d), 169.6 (s). Anal. Calcd for $\mathrm{C}_{13} \mathrm{H}_{16} \mathrm{O}_{2}$ : C, 76.44; H, 7.90. Found: C, 76.38; H, 7.81.

\section{4-(bicyclo[2.2.1]hept-5-en-2-yl)but-3-ynyl acetate $(4 \mathbf{j})$}

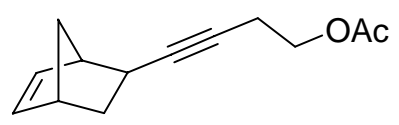

4j

Chemical yield : 91\%. Colorless oil. $R f\left(\mathrm{PE}_{\mathrm{Et}} \mathrm{O}\right.$, 95/5) 0.43; IR (neat) v 3062, 2233, 1746, $1573 \mathrm{~cm}^{-1}$; ${ }^{1} \mathrm{H}$ NMR $\left(\mathrm{CDCl}_{3}, 200 \mathrm{MHz}\right) \delta 1.50$ (m, 4H), 2.02 (m, 1H), 2.03 (s, 3H), 2.47 (dt, $J=2.0,7.0 \mathrm{~Hz}, 2 \mathrm{H}), 2.83$ (m, 2H), 4.11 (t, $J=7.0 \mathrm{~Hz}, 2 \mathrm{H}), 6.01$ (m, 2H); ${ }^{13} \mathrm{C} \mathrm{NMR}\left(\mathrm{CDCl}_{3}, 50 \mathrm{MHz}\right) \delta 19.3(\mathrm{t}), 20.9(\mathrm{q}), 28.8(\mathrm{~d}), 34.7$ (t), 41.8 (d), $46.6(\mathrm{t}), 49.2$ 
(d), 62.8 (t), 75.1 (s), 86.8 (s), 135.2 (d), 137.2 (d), 170.8 (s). Anal. Calcd for $\mathrm{C}_{13} \mathrm{H}_{16} \mathrm{O}_{2}$ : C, 76.44; H, 7.90. Found: C, 76.58; H, 7.78.

\section{3-(bicyclo[2.2.1]hept-5-en-2-yl)prop-2-ynyl methyl carbonate (4k)}

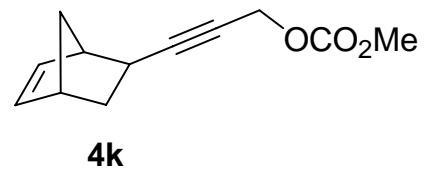

Chemical yield : 70\%. Colorless oil. $R f\left(\mathrm{PE} / \mathrm{Et}_{2} \mathrm{O}, 95 / 5\right)$ 0.46; IR (neat) v 3066, 2234, 1756, $1263 \mathrm{~cm}^{-1} ;{ }^{1} \mathrm{H}$ NMR $\left(\mathrm{CDCl}_{3}, 200 \mathrm{MHz}\right) \delta 1.49$ (m, 3H), 1.65 (dt, $J=3.5,11.3$ Hz, 1H), 2.12 (m, 1H), 2.88 (m, 2H), 3.78 (s, 3H), 4.71 (d, $J=2.1 \mathrm{~Hz}, 2 \mathrm{H}), 6.01$ (m, 2H); ${ }^{13} \mathrm{C} \mathrm{NMR}\left(\mathrm{CDCl}_{3}, 50 \mathrm{MHz}\right) \delta 28.9$ (d), 34.4 (t), 41.8 (d), 46.7 (t), 48.9 (d), 55.0 (d), 56.4 (q), 73.0 (s), 93.0 (s), 135.1 (d), 137.4 (d), 154.6 (s). Anal. Calcd for $\mathrm{C}_{12} \mathrm{H}_{14} \mathrm{O}_{3}$ : C, 69.89; H, 6.84. Found: C, 69.68; H, 6.71.

dimethyl 2-(3-(bicyclo[2.2.1]hept-5-en-2-yl)prop-2-ynyl)propanedioate (4l)

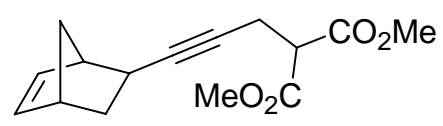

4l

Chemical yield : 92\%. Colorless oil. $R f\left({\left.\mathrm{PE} / \mathrm{Et}_{2} \mathrm{O}, 80 / 20\right)}^{0.48}\right.$; IR (neat) v 2955, 2232, 1755, 1740, 1437, $1240 \mathrm{~cm}^{-1}$; ${ }^{1} \mathrm{H}$ NMR $\left(\mathrm{CDCl}_{3}, 200 \mathrm{MHz}\right) \delta$ 1.36-1.62 (m, 4H), 2.05 (m, 1H), 2.76 (dd, $J=2.2,7.8 \mathrm{~Hz}, 2 \mathrm{H}$ ), 2.81 (br, 1H), 2.86 (br, $1 \mathrm{H}), 3.57$ (t, $J=7.8 \mathrm{~Hz}$, 1H), 3.77 (s, 6H), 5.99 (dd, $J=2.7,8.2 \mathrm{~Hz}, 1 \mathrm{H}$ ), 6.04 (dd, $J=2.7,8.3 \mathrm{~Hz}, 1 \mathrm{H}$ ); ${ }^{13} \mathrm{C}$ NMR (CDCl, $50 \mathrm{MHz}) \delta 18.9$ (t), 28.6 (d), 34.5 (t), 41.6 (d), 46.4 (t), 49.1 (d), 51.5 (d), 52.4 (q), 75.0 (s), 87.4 (s), 135.0 (d), 137.1 (d), 168.4 (s). Anal. Calcd for $\mathrm{C}_{15} \mathrm{H}_{18} \mathrm{O}_{4}$ : C, 68.69; H, 6.92. Found: C, 68.47; H, 7.02.

diethyl (2-propenyl)(3-(bicyclo[2.2.1]hept-5-en-2-yl)prop-2-ynyl)propanedioate $(4 \mathrm{~m})$

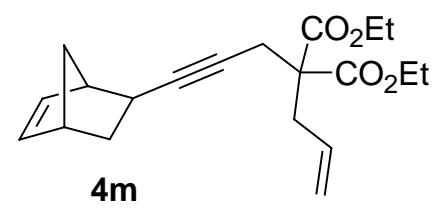

Chemical yield : 82\%. Colorless oil. $R f\left(\mathrm{PE} / \mathrm{Et}_{2} \mathrm{O}, 95 / 5\right)$ 0.43; IR (neat) v 2232, 1735, 1642, $709 \mathrm{~cm}^{-1}$; ${ }^{1} \mathrm{H}$ NMR $\left(\mathrm{CDCl}_{3}, 200 \mathrm{MHz}\right) \delta 1.23$ (t, $\left.J=7.1 \mathrm{~Hz}, 6 \mathrm{H}\right), 1.36-1.62$ (m, 4H), 2.04 (m, 1H), 2.68-2.90 (m, 6H), 4.16 (q, $J=7.1$ Hz, 4H), 5.01-5.20 (m, 2H), 5.62 (ddt, $J=17.2,9.8,7.4 \mathrm{~Hz}, 1 \mathrm{H}), 5.99(\mathrm{~m}, 2 \mathrm{H}) ;{ }^{13} \mathrm{C} \mathrm{NMR}\left(\mathrm{CDCl}_{3}, 50 \mathrm{MHz}\right) \delta 14.0$ (q), $22.8(\mathrm{t}), 28.7(\mathrm{~d}), 34.7(\mathrm{t}), 36.3(\mathrm{t}), 41.7(\mathrm{~d}), 46.5(\mathrm{t}), 49.2(\mathrm{~d}), 57.0(\mathrm{~s}), 61.3(\mathrm{t}), 74.0(\mathrm{~s})$, 
88.3 (s), 119.3 (t), 132.0 (d), 135.1 (d), 137.1 (d), 169.9 (s). Anal. Calcd for $\mathrm{C}_{20} \mathrm{H}_{26} \mathrm{O}_{4}$ : C, 72.70; H, 7.93. Found: C, 72.57; H, 7.78.

7-(bicyclo[2.2.1]hept-5-en-2-yl)-4,4-dimethylhept-6-yn-2-one (4n)

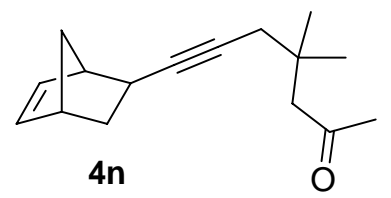

Chemical yield : 90\%. Colorless oil. $R f\left(\mathrm{PE}_{\mathrm{Et}} \mathrm{O}, 95 / 5\right)$ 0.38; IR (neat) v 2226, 1718, 1364, 1158, $708 \mathrm{~cm}^{-1} ;{ }^{1} \mathrm{H}$ NMR $\left(\mathrm{CDCl}_{3}, 200 \mathrm{MHz}\right) \delta 1.03$ (s, 3H), 1.10 (m, 2H), 1.51 (m, 4H), 2.04-2.25 (m, 3H), 2.12 (s, 3H), 2.42 (s, 2H), 2.46 (m, 1H), 2.85 (m, 2H), 5.956.12 (m, 2H); ${ }^{13} \mathrm{C} \mathrm{NMR}\left(\mathrm{CDCl}_{3}, 50 \mathrm{MHz}\right) \delta 27.0$ (q), 28.8 (d), 31.9 (t), 32.1 (q), 33.7 (s), 34.8 (t), 47.1 (d), 46.6 (t), 49.3 (d), 52.5 (t), 77.2 (s), 87.3 (s), 135.2 (d), 137.0 (d), 208.4 (s). Anal. Calcd for $\mathrm{C}_{16} \mathrm{H}_{22} \mathrm{O}$ : C, 83.43; H, 9.63. Found: C, 83.31; H, 9.49.

(3-(bicyclo[2.2.1]hept-5-en-2-yl)prop-2-ynyl)(phenyl)sulfane (40) was obtained along with prop-2-ynylsulfanyl-benzene (3o) as an inseparable 1:1 mixture.

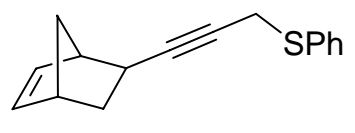

40

Chemical yield : 37\%. $R f(\mathrm{PE})$ 0.55; ${ }^{13} \mathrm{C} \mathrm{NMR}\left(\mathrm{CDCl}_{3}, 50 \mathrm{MHz}\right) \delta 23.2$ (t), 28.8 (d), 34.4 (t), 41.7 (d), 46.5 (t), 49.0 (d), 75.1 (s), 88.9 (s), 126.4 (d), 128.6 (d), 129.9 (d), $135.0(\mathrm{~s}+\mathrm{d}), 137.1(\mathrm{~d})$.

\section{5-exo-(3-(phenylsulfonyl)prop-1-ynyl)bicyclo[2.2.1]hept-2-ene (4p)}

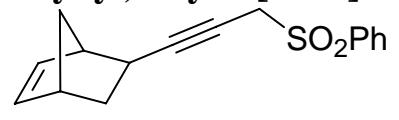

$4 p$

Chemical yield : 52\%. Colorless oil. $R f$ (PE/AcOEt, 80/20) 0.61; IR (neat) v 2974, 2237, 1593, 1455, 1326, $1136 \mathrm{~cm}^{-1} ;{ }^{1} \mathrm{H}$ NMR $\left(\mathrm{CDCl}_{3}, 200 \mathrm{MHz}\right) \delta 1.37$ (m, 3H), 1.52 (dt, $J=$ 11.5, 3.9 Hz, 1H), 2.03 (m, 1H), 2.77 (m, 1H), 2.84 (m, 1H), 3.94 (d, $J=2.3 \mathrm{~Hz}, 2 \mathrm{H}$ ), 5.95 (dd, $J=3.0,5.6 \mathrm{~Hz}, 1 \mathrm{H}$ ), 6.03 (dd, $J=2.9,5.6 \mathrm{~Hz}, 1 \mathrm{H}), 7.61$ (m, 3H), 7.94 (m, $2 \mathrm{H}) ;{ }^{13} \mathrm{C} \mathrm{NMR}\left(\mathrm{CDCl}_{3}, 50 \mathrm{MHz}\right) \delta 28.8$ (d), 34.1 (t), 41.7 (d), 46.6 (t), 48.8 (d), 49.1 (t), 67.2 (s), 93.3 (s), 128.8 (d), 128.9 (d), 134.0 (d), 134.9 (d), 137.4 (d), 137.7 (s). Anal. Calcd for $\mathrm{C}_{16} \mathrm{H}_{16} \mathrm{SO}_{2}$ : C, 70.56; H, 5.92. Found: C, 70.42; H, 5.81 . 


\section{3-(bicyclo[2.2.1]hept-5-en-2-yl)prop-2-yn-1-ol (4q)}

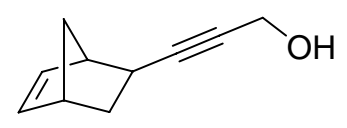

$4 q$

Chemical yield : 44\%. Colorless oil. Rf (PE/AcOEt, 85/15) 0.37; IR (neat) v 3350, 2225, 1342, 1132, 1023, $709 \mathrm{~cm}^{-1}$; ${ }^{1} \mathrm{H}$ NMR $\left(\mathrm{CDCl}_{3}, 200 \mathrm{MHz}\right) \delta 1.53$ (m, 5H), 2.13 (m, 1H), 2.87 (m, 2H), 4.23 (dd, $J=2.0,5.8 \mathrm{~Hz}, 2 \mathrm{H}), 6.01(\mathrm{~m}, 2 \mathrm{H}) ;{ }^{13} \mathrm{C} \mathrm{NMR}\left(\mathrm{CDCl}_{3}, 50\right.$ MHz) $\delta 28.8$ (d), 34.5 (t), 41.8 (d), 46.6 (t), 49.0 (d), 51.3 (t), 78.0 (s), 91.0 (s), 135.1 (d), 137.3 (d). Anal. Calcd for $\mathrm{C}_{10} \mathrm{H}_{12} \mathrm{O}$ : C, 81.04; H, 8.16. Found: C, 80.87; H, 8.02.

\section{4-(bicyclo[2.2.1]hept-5-en-2-yl)but-3-yn-1-ol (4r)}

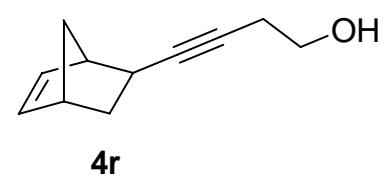

Chemical yield : 52\%. Colorless oil. $R f(\mathrm{PE} / \mathrm{AcOEt}, 85 / 15)$ 0.33; IR (neat) v 3340, 2977, $2232 \mathrm{~cm}^{-1}$; ${ }^{1} \mathrm{H} \mathrm{NMR}\left(\mathrm{CDCl}_{3}, 200 \mathrm{MHz}\right) \delta 1.44$ (m, 3H), 1.60 (dd, $\left.J=11.2,3.8 \mathrm{~Hz}, 1 \mathrm{H}\right)$, 1.79 (m, 1H), 2.08 (m, 1H), 2.42 (dd, $J=2.2,6.2$ Hz, 2H), 2.83 (m, 2H), 3.62 (m, 2H), $6.01(\mathrm{~m}, 2 \mathrm{H}) ;{ }^{13} \mathrm{C} \mathrm{NMR}\left(\mathrm{CDCl}_{3}, 50 \mathrm{MHz}\right) \delta 23.3(\mathrm{t}), 28.8(\mathrm{~d}), 34.8(\mathrm{t}), 41.8(\mathrm{~d}), 46.6(\mathrm{t})$, 49.3 (d), 61.4 (t), 75.9 (s), 87.5 (s), 135.3 (d), 137.2 (d). Anal. Calcd for $\mathrm{C}_{11} \mathrm{H}_{14} \mathrm{O}: \mathrm{C}$, 81.44; H, 8.70. Found: C, 81.28; H, 8.54.

\section{1-(2-(bicyclo[2.2.1]hept-5-en-2-yl)ethynyl)cyclohexanol (4s)}

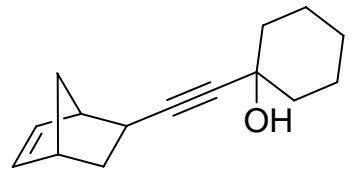

$4 s$

Chemical yield : 97\%. White solid. Mp 59-60 ${ }^{\circ} \mathrm{C} ; R f\left(\mathrm{PE} / \mathrm{Et}_{2} \mathrm{O} 95 / 5\right)$ 0.20; IR (KBr) v 3276, 3065, 2977, 2228, $1621 \mathrm{~cm}^{-1}$; ${ }^{1} \mathrm{H}$ NMR $\left(\mathrm{CDCl}_{3}, 200 \mathrm{MHz}\right) \delta 1.20$ (m, 1H), 1.38$1.73(\mathrm{~m}, 12 \mathrm{H}), 1.84(\mathrm{~m}, 1 \mathrm{H}), 2.12(\mathrm{~m}, 1 \mathrm{H}), 2.86(\mathrm{~m}, 2 \mathrm{H}), 6.01(\mathrm{~m}, 2 \mathrm{H}) ;{ }^{13} \mathrm{C} \mathrm{NMR}$ $\left(\mathrm{CDCl}_{3}, 50 \mathrm{MHz}\right) \delta 23.5(\mathrm{t}), 25.2(\mathrm{t}), 28.7(\mathrm{~d}), 34.9(\mathrm{t}), 40.27$ (t), 40.32 (t), $41.8(\mathrm{~d}), 46.7$ 
(d), 49.2 (d), 68.8 (s), 83.5 (s), 89.6 (s), 135.2 (d), 137.2 (d). Anal. Calcd for $\mathrm{C}_{15} \mathrm{H}_{20} \mathrm{O}$ : C, 83.29; H, 9.32. Found: C, 83.32; H, 9.25.

\section{4-(3-(bicyclo[2.2.1]hept-5-en-2-yl)prop-2-ynyl)morpholine (4t)}

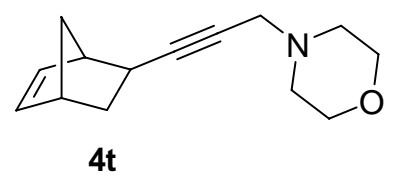

Chemical yield : 69\%. Colorless oil. $R f\left(\mathrm{Et}_{2} \mathrm{O}\right)$ 0.59; IR (neat) $v 2970,2231,1574,1460$, 1338, $1122 \mathrm{~cm}^{-1}$; ${ }^{1} \mathrm{H}$ NMR $\left(\mathrm{CDCl}_{3}, 200 \mathrm{MHz}\right) \delta$ 1.38-1.68 (m, 4H), 2.11 (m, 1H), 2.52 (pseudo t, $J=4.6 \mathrm{~Hz}, 4 \mathrm{H}$ ), 2.86 (m, 2H), 3.24 (d, $J=2.0 \mathrm{~Hz}, 2 \mathrm{H}$ ), 3.72 (pseudo t, $J=$ $4.6 \mathrm{~Hz}, 4 \mathrm{H}), 6.02$ (m, 2H); ${ }^{13} \mathrm{C} \mathrm{NMR}\left(\mathrm{CDCl}_{3}, 50 \mathrm{MHz}\right) \delta 28.8$ (d), 34.7 (t), 41.8 (d), 46.7 (t), 47.7 (t), 49.2 (d), 52.2 (t), 66.8 (t), 74.0 (s), 90.6 (s), 135.2 (d), 137.1 (d). Anal. Calcd for $\mathrm{C}_{14} \mathrm{H}_{19} \mathrm{NO}$ : C, 77.38; H, 8.81; N, 6.45. Found: C, 77.17; H, 8.65; N, 6.37.

\section{(E)-hex-2-en-4-yne-1,6-diol (5)}

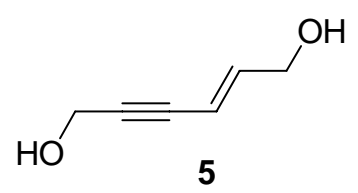

Chemical yield : 43\%. White solid. Mp 48-49 ${ }^{\circ} \mathrm{C} ; R f$ (PE/AcOEt, 40/60) 0.47; ${ }^{1} \mathrm{H}$ NMR $\left(\mathrm{CD}_{3} \mathrm{COCD}_{3} / \mathrm{D}_{2} \mathrm{O}, 200 \mathrm{MHz}\right) \delta 3.35$ (br s, 2H), 4.08 (d, $\left.J=4.5 \mathrm{~Hz}, 2 \mathrm{H}\right), 4.23$ (br s, 2H), $5.75(\mathrm{dm}, J=15.9 \mathrm{~Hz}, 1 \mathrm{H}), 6.18(\mathrm{dt}, J=15.9,4.5 \mathrm{~Hz}, 1 \mathrm{H}) ;{ }^{13} \mathrm{C} \mathrm{NMR}\left(\mathrm{CDCl}_{3}, 50 \mathrm{MHz}\right)$ $\delta 46.6(\mathrm{t}), 62.3(\mathrm{t}), 83.3$ (s), 88.0 (s), 109.3 (d), 142.4 (d).

Trimethyl-(1,2,3,4-tetrahydro-1,4-methano-naphthalen-2ylethynyl)-silane (8)

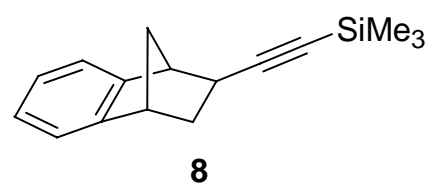

Chemical yield : 69\%. Colorless oil that solidified on standing. $\mathrm{Mp} 25^{\circ} \mathrm{C}$; $R f(\mathrm{PE}) 0.53$; IR (KBr) v 3021, 2171, 1943, 1867, $1802 \mathrm{~cm}^{-1}$; ${ }^{1} \mathrm{H}$ NMR $\left(\mathrm{CDCl}_{3}, 200 \mathrm{MHz}\right) \delta 0.17$ (s, 9H), 1.71 (m, 2H), 1.96 (m, 2H), 2.31 (dd, $J=8.7,4.3$ Hz, 1H), 3.37 (m, 1H), 3.42 (m, 1H), 7.12 (m, 4H); ${ }^{13} \mathrm{C}$ NMR $\left(\mathrm{CDCl}_{3}, 50 \mathrm{MHz}\right) \delta 0.2$ (qx3), 31.9 (d), 37.2 (t), 43.7 (d), 47.5 (t), 50.9 (d), 84.5 (s), 111.6 (s), 120.7 (d), 121.1 (d), 125.7 (d), 126.2 (d), 146.6 (s), 147.9 (s). Anal. Calcd for $\mathrm{C}_{16} \mathrm{H}_{20} \mathrm{Si}$ : C, 79.93; H, 8.38. Found: C, 79.80; H, 8.27. 


\section{Trimethyl-(11-oxa-tricyclo[6.2.1.0 $\left.{ }^{2,7}\right]$ undeca-2,4,6-trien-9-ylethynyl)-silane (9)}

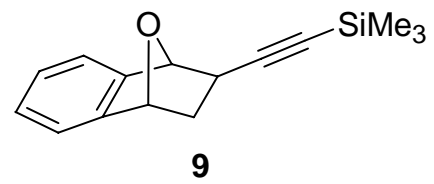

Chemical yield : 97\%. White solid. Mp 99-101 C; Rf (PE/EtOAc, 95/5) 0.65; IR (KBr) v 3051, 2168, 1961, 1919, 1888, $1819 \mathrm{~cm}^{-1}$; ${ }^{1} \mathrm{H}$ NMR $\left(\mathrm{CDCl}_{3}, 200 \mathrm{MHz}\right) \delta 0.24$ (s, 9H), 1.92 (dd, $J=11.5,8.5 \mathrm{~Hz}, 1 \mathrm{H}$ ), 2.24 (ddd, $J=11.5,4.8,4.4 \mathrm{~Hz}, 1 \mathrm{H}$ ), 2.61 (dd, $J=8.5$, $4.2 \mathrm{~Hz}, 1 \mathrm{H}), 5.45$ (m, 1H), 5.55 (d, $J=4.9 \mathrm{~Hz}, 1 \mathrm{H}), 7.20-7.34$ (m, 4H); ${ }^{13} \mathrm{C}$ NMR $\left(\mathrm{CDCl}_{3}, 50 \mathrm{MHz}\right) \delta 0.1$ (qx3), 32.5 (d), 36.6 (t), 79.0 (d), 84.2 (d), 85.1 (s), 108.8 (s), 118.9 (d), 119.1 (d), 126.7 (d), 127.0 (d), 144.3 (s), 145.1 (s). Anal. Calcd for $\mathrm{C}_{15} \mathrm{H}_{18} \mathrm{OSi}$ : C, 74.33; H, 7.49. Found: C, 74.26; H, 7.42.

\section{3-Methoxycarbonyloxymethyl-bicyclo[2.2.1]hepta-2,5-dien-2-ylmethyl,}

methyl carbonate (10)

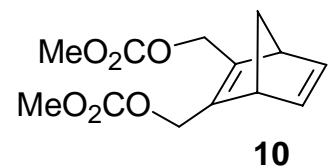

Methyl chloroformate $(0.39 \mathrm{~mL}, 5.1 \mathrm{mmol})$ was added dropwise to an ice cooled solution of (3-hydroxymethyl-bicyclo[2.2.1] hepta-2,5-dien-2-yl)-methanol ${ }^{5}$ (190 mg, $1.27 \mathrm{mmol}$ ), pyridine (0.8 g, $0.82 \mathrm{~mL}, 10.1 \mathrm{mmol})$ and DMAP (10.0 mg, $0.08 \mathrm{mmol})$ in $\mathrm{CH}_{2} \mathrm{Cl}_{2}(7 \mathrm{~mL})$. The resulting mixture was stirred at $0^{\circ} \mathrm{C}$ for $15 \mathrm{~min}$, then at room temperature for $12 \mathrm{~h}$. The reaction mixture was concentrated in vacuo and diluted by addition of water $(1 \mathrm{~mL})$ and ether $(5 \mathrm{~mL})$. The organic layer was washed with a saturated aqueous solution of $\mathrm{CuSO}_{4}(3 \times 1 \mathrm{~mL})$, brine $(2 \mathrm{~mL})$ and dried over $\mathrm{Na}_{2} \mathrm{SO}_{4}$. After evaporation of the solvent, the crude product was purified by column chromatography (PE/Et $\mathrm{H}_{2} \mathrm{O}$ 1/9) to afford $300 \mathrm{mg}$ (88\%) of $\mathbf{1 0}$ as a pale yellow oil.

$\mathrm{R}_{f}\left(\mathrm{PE} / \mathrm{Et}_{2} \mathrm{O}\right.$ 1/1) 0.58. IR (neat) $v$ 2961, 2869, 1746, 1445, 1374, 1254, $939 \mathrm{~cm}^{-1} .{ }^{1} \mathrm{H}$ NMR (200 MHz, $\left.\mathrm{CDCl}_{3}\right) \delta 1.94(\mathrm{dm}, J=6.2 \mathrm{~Hz}, 1 \mathrm{H}), 2.04(\mathrm{dm}, J=5.8 \mathrm{~Hz}, 1 \mathrm{H}), 3.57$ (m, 2H), 3.78 (s, 6H), 4.72 (d, $J=12.4 \mathrm{~Hz}, 2 \mathrm{H}), 4.87$ (d, $J=13.2 \mathrm{~Hz}, 2 \mathrm{H}), 6.78$ (app t, $J$ $=1.9 \mathrm{~Hz}, 2 \mathrm{H},) .{ }^{13} \mathrm{C}$ NMR $\left(50 \mathrm{MHz}, \mathrm{CDCl}_{3}\right) \delta 52.4(\mathrm{q}), 54.7(\mathrm{~d}), 63.5(\mathrm{t}), 71.8(\mathrm{t}), 142.3$ (d), 148.2 (s), 155.6 (s). Anal. Calcd for $\mathrm{C}_{13} \mathrm{H}_{16} \mathrm{O}_{6}$ : C, 58.20; H, 6.01. Found: C, 58.04; H, 5.93 . 


\section{Cyclopropane 12}

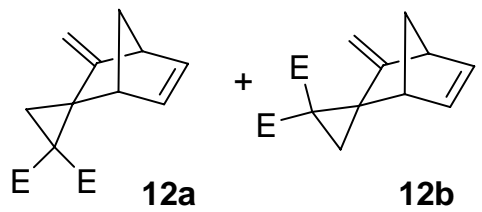

Biscarbonate 10 (134.1 mg, $0.5 \mathrm{mmol}$ ) was added to a solution of palladium tetrakistriphenylphosphine (28 mg, $0.025 \mathrm{mmol}$ ) and methyl malonate 11 (66 mg, 0.55 $\mathrm{mmol})$ in THF (2.5 mL) and the mixture was stirred at $50^{\circ} \mathrm{C}$ for $18 \mathrm{~h}$. After removal of the solvent under vacuum, the diasteromeric ratio 12a/12b (87/13) was determined by NMR analysis. The crude material was purified by silica gel chromatography (elution with $\left.\mathrm{Et}_{2} \mathrm{O} / \mathrm{PE} 1 / 9\right)$ to afford $95.5 \mathrm{mg}$ (77\%) of 12 as a colorless oil.

Spectroscopic data are in agreement with those reported in ref. 6.

\section{3-Methoxycarbonyloxymethyl-6-phenylethynyl-bicyclo[2.2.1]hept-2-en-2-ylmethyl,} methyl carbonate (13)

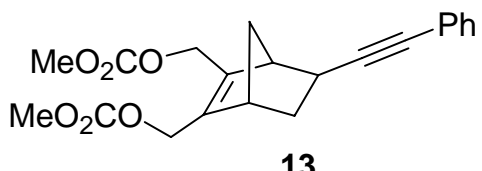

13

As reported for the general procedure, the reaction of an equimolar mixture of biscarbonate 10, phenylethyne (3a) and methyl malonate $\mathbf{1 1}$ in the presence of phosphapalladacycle 1 (2.5 mol\%) in DCE for $1 \mathrm{~h}$ at $50^{\circ} \mathrm{C}$ afforded after colum chromatography (elution with $\mathrm{PE} / \mathrm{Et}_{2} \mathrm{O} 4 / 1$ ) enyne 13 as a colorless oil in $88 \%$ yield. $R f\left(\mathrm{PE} / \mathrm{Et}_{2} \mathrm{O}, 70 / 30\right)$ 0.34; IR (neat) v 2961, 2222, 1748, 1442, 1263, $788 \mathrm{~cm}^{-1} ;{ }^{1} \mathrm{H} \mathrm{NMR}$ $\left(\mathrm{CDCl}_{3}, 300 \mathrm{MHz}\right) \delta 1.60(\mathrm{dt}, J=1.9,8.7 \mathrm{~Hz}, 1 \mathrm{H}), 1.65(\mathrm{dt}, J=2.2,11.5 \mathrm{~Hz}, 1 \mathrm{H}), 1.73$ (d, $J=8.9 \mathrm{~Hz}, 1 \mathrm{H}$ ), 1.89 (dt, $J=3.8,11.7 \mathrm{~Hz}, 1 \mathrm{H}$ ), 2.47 (ddd, $J=1.0,4.7,8.7 \mathrm{~Hz}, 1 \mathrm{H}$ ), 3.03 (m, 1H), 3.10 (m, 1H), 3.79 (s, 3H), 3.80 (s, 3H), 4.72 (app dd, $J=7.6,13.0 \mathrm{~Hz}$, 2H), 4.82 (app d, $J=13.0 \mathrm{~Hz}, 2 \mathrm{H}), 7.24-7.30$ (m, 3H), 7.35-7.40 (m, 2H); ${ }^{13} \mathrm{C}$ NMR $\left(\mathrm{CDCl}_{3}, 50 \mathrm{MHz}\right) \delta 30.1$ (d), 35.2 (t), 44.4 (d), 45.4 (t), 51.8 (d), 54.7 (q), $62.2(\mathrm{t}), 62.3$ (t), 80.5 (s), 93.8 (s), 123.7 (s), 127.4 (d), 128.0 (d), 131.3 (d), 139.8 (s), 142.3 (s), 155.5 (s). Anal. Calcd for $\mathrm{C}_{21} \mathrm{H}_{22} \mathrm{O}_{6}$ : C, 68.10; H, 5.99. Found: C, 67.86; H, 5.81. 


\section{$\left(2 R^{*}, 3 S^{*}, 5 S^{*}\right)$-5-Phenylethynyl-bicyclo[2.2.1]heptane-2,3-diol}

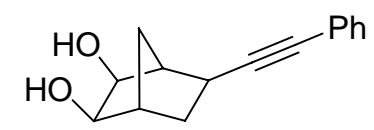

Osmium tetroxide (ca. $5 \mathrm{mg}, 2.10^{-2} \mathrm{mmol}$ ) was added to an ice-cooled mixture of exo-5phenylethynyl-bicyclo[2.2.1]hept-2-ene (4a) (97 mg, $0.5 \mathrm{mmol})$ and NMO (67.5 mg, $0.5 \mathrm{mmol})$ in a (8/1) mixture of THF/water $(2 \mathrm{~mL})$ The mixture was stirred for $5.5 \mathrm{~h}$ at room temperature until complete consumption of the enyne monitored by TLC. The solution was treated by aqueous hydrochloric acid $2 \mathrm{~N}(4 \mathrm{~mL})$, aqueous sodium hydrogenosulfite $15 \%(1.5 \mathrm{~mL})$ and AcOEt $(4 \mathrm{~mL})$. The aqueous layer was extracted by AcOEt $(3 \times 4 \mathrm{~mL})$ and the combined organic phases were dried over $\mathrm{Na}_{2} \mathrm{SO}_{4}$ and concentrated under vacuo. The crude product was purified by silica gel chromatography (elution with 40-50\% AcOEt in hexanes) to afford $72 \mathrm{mg}$ (63 \%) of pure diol as a white solid.

Mp 86-87 C; Rf (AcOEt/PE 1/1) 0.50; IR (KBr) v 3432, 3387, 3240, 2966, 2950, 2877, 2225, $755 \mathrm{~cm}^{-1}$; ${ }^{1} \mathrm{H}$ NMR $\left(\mathrm{CDCl}_{3}, 200 \mathrm{MHz}\right) \delta$ 1.53-1.67 (m, 2H), 1.68 (dt, $J=4.9,10.9$ Hz, 1H), 1.85 (br d, $J=10.9$ Hz, 1H), 2.25 (m, 1H), 2.35-2.42 (m, 2H), 2.64 (m, 1H, $\mathrm{OH}), 2.71$ (m, 1H, OH), 3.72 (m, 2H), 7.25-7.29 (m, 3H), 7.34-7.39 (m, 2H); ${ }^{13} \mathrm{C}$ NMR $\left(\mathrm{CDCl}_{3}, 50 \mathrm{MHz}\right) \delta 29.1$ (d), 30.1 (t), 34.3 (t), 42.8 (d), 50.0 (d), 73.5 (d), 73.9 (d), 80.7 (s), 93.6 (s), 123.7 (s), 127.6 (d), 128.1 (d), 131.4 (d). Anal. Calcd for $\mathrm{C}_{15} \mathrm{H}_{16} \mathrm{O}_{2}$ : C, 78.92; H, 7.06. Found: C, 78.69; H, 7.13.

$\left(1 S^{*}, 3 S^{*}, 4 S^{*}\right)-4-P h e n y l e t h y n y l-c y c l o p e n t a n e-1,3-d i c a r b a l d e h y d e ~(14)$

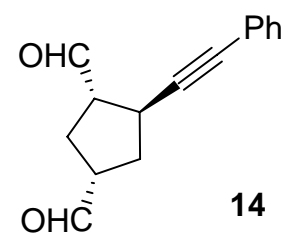

Sodium metaperiodate $(128 \mathrm{mg}, 0.6 \mathrm{mmol})$ was added portionwise to a mixture of saturated aqueous $\mathrm{NaHCO}_{3}(50 \mu \mathrm{L})$ and a solution of the above diol (68.4 mg, 0.3 mmol) in $\mathrm{CH}_{2} \mathrm{Cl}_{2}(1 \mathrm{~mL})$. The resulting mixture was vigorously stirred at room temperature for $1.5 \mathrm{~h}$. The organic phase was removed and the residue was extracted 
with $\mathrm{CH}_{2} \mathrm{Cl}_{2}(3 \times 0.5 \mathrm{~mL})$. The combined organic phases were dried over $\mathrm{Na}_{2} \mathrm{SO}_{4}$ and evaporated under vacuo to afford quantitatively the title compound as a colorless oil.

Rf (AcOEt/PE 1/1) 0.58; IR (neat) v 3051, 2960, 2874, 2219, $1720733 \mathrm{~cm}^{-1}$; ${ }^{1} \mathrm{H}$ NMR

$\left(\mathrm{CDCl}_{3}, 300 \mathrm{MHz}\right) \delta 2.13$ (ddd, $\left.J=4.8,7.9,13.2 \mathrm{~Hz}, 1 \mathrm{H}\right), 2.25-2.42$ (m, 3H), 3.03-3.18

(m, 2H), 3.23 (app q, $J=7.3 \mathrm{~Hz}, 1 \mathrm{H}), 7.28-7.32$ (m, 3H), 7.38-7.42 (m, 2H), 9.70 (s, 1H), 9.76 (s, 1H); ${ }^{13} \mathrm{C} \mathrm{NMR}\left(\mathrm{CDCl}_{3}, 50 \mathrm{MHz}\right) \delta 25.0$ (t), 31.2 (d), 33.4 (t), 49.7 (d), 58.0 (d), 82.2 (s), 89.6 (s), 122.9 (s), 128.1 (d), 128.2 (d), 131.5 (d), 200.5 (s), 201.4 (s). Anal. Calcd for $\mathrm{C}_{15} \mathrm{H}_{14} \mathrm{O}_{2}$ : C, 79.62; H, 6.24. Found: C, 79.19; H, 6.40.

\section{- References}

(1) Brandsma, L. Preparative Acetylenic Chemistry; Elsevier: Amsterdam, 2nd Ed., 1988.

(2) Tsuji, J.; Sato, K.; Okumoto, H. J. Org. Chem. 1984, 49, 1341.

(3) Felix, D.; Schreiber, J.; Ohloff, G.; Eschenmoser, A. Helv. Chim. Acta 1971, 54, 2896.

(4) Stirling, C. J. M. J. Chem. Soc. 1964, 5856.

(5) Ranchoux, M.; Brunel, J. M.; Iacazio, G.; Buono, G Tetrahedron Asymmetry 1998, 9, 581.

(6) Brunel, J. M.; Maffei, M.; Muchow, G.; Buono, G. Eur. J. Org. Chem. 2001, 1009. 
- NMR Spectra of compounds (4a) and D-(4a)
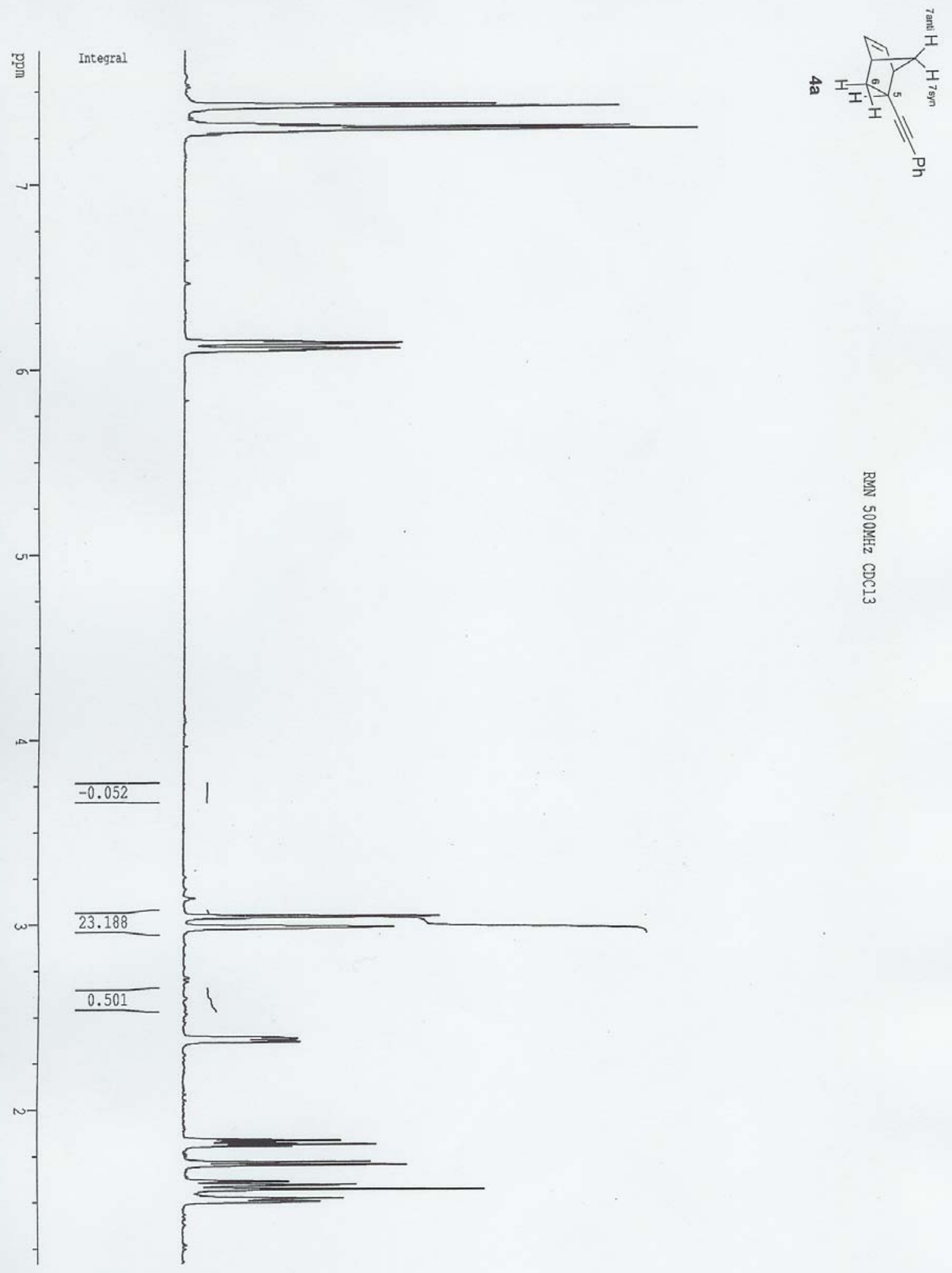

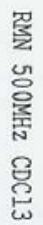




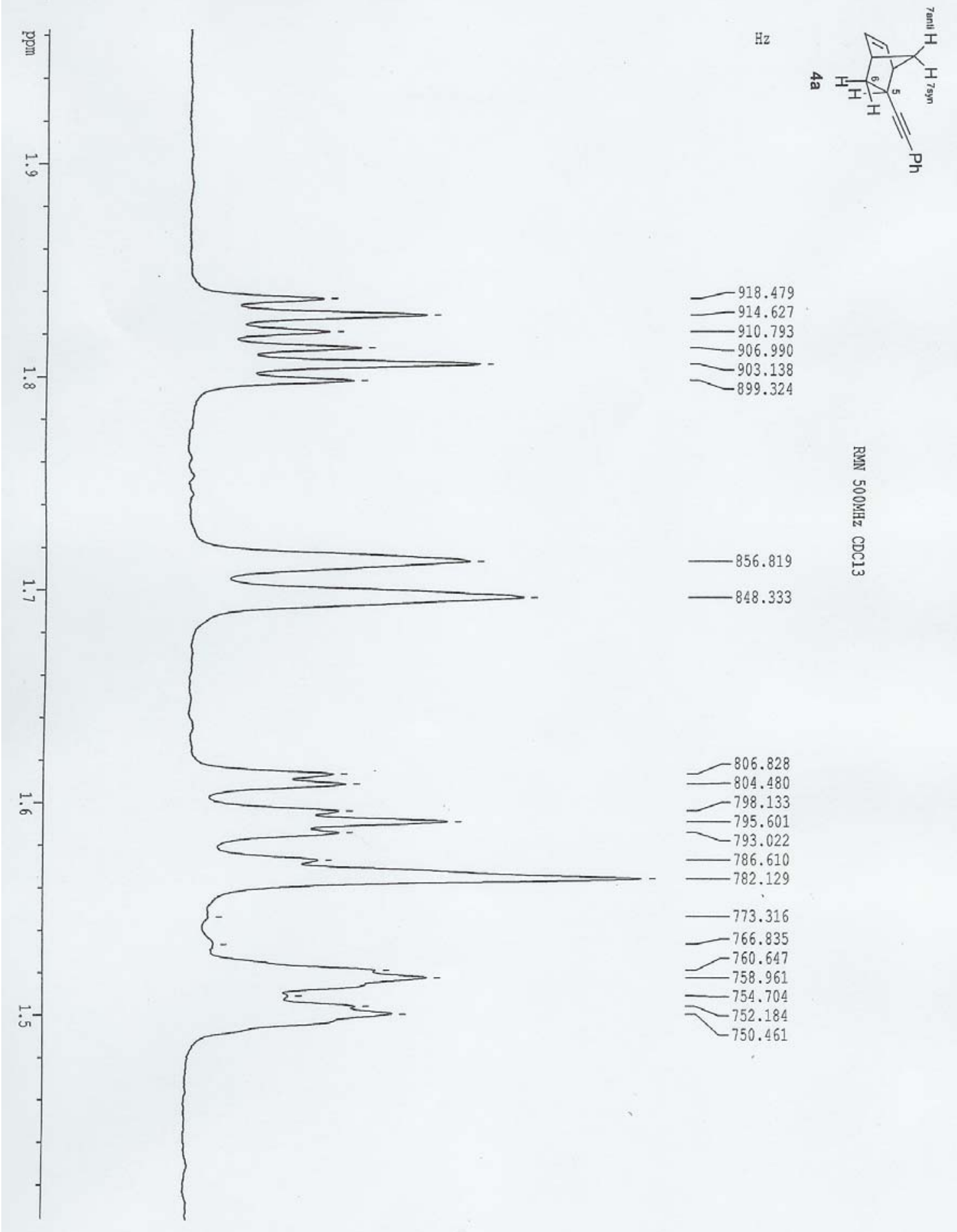




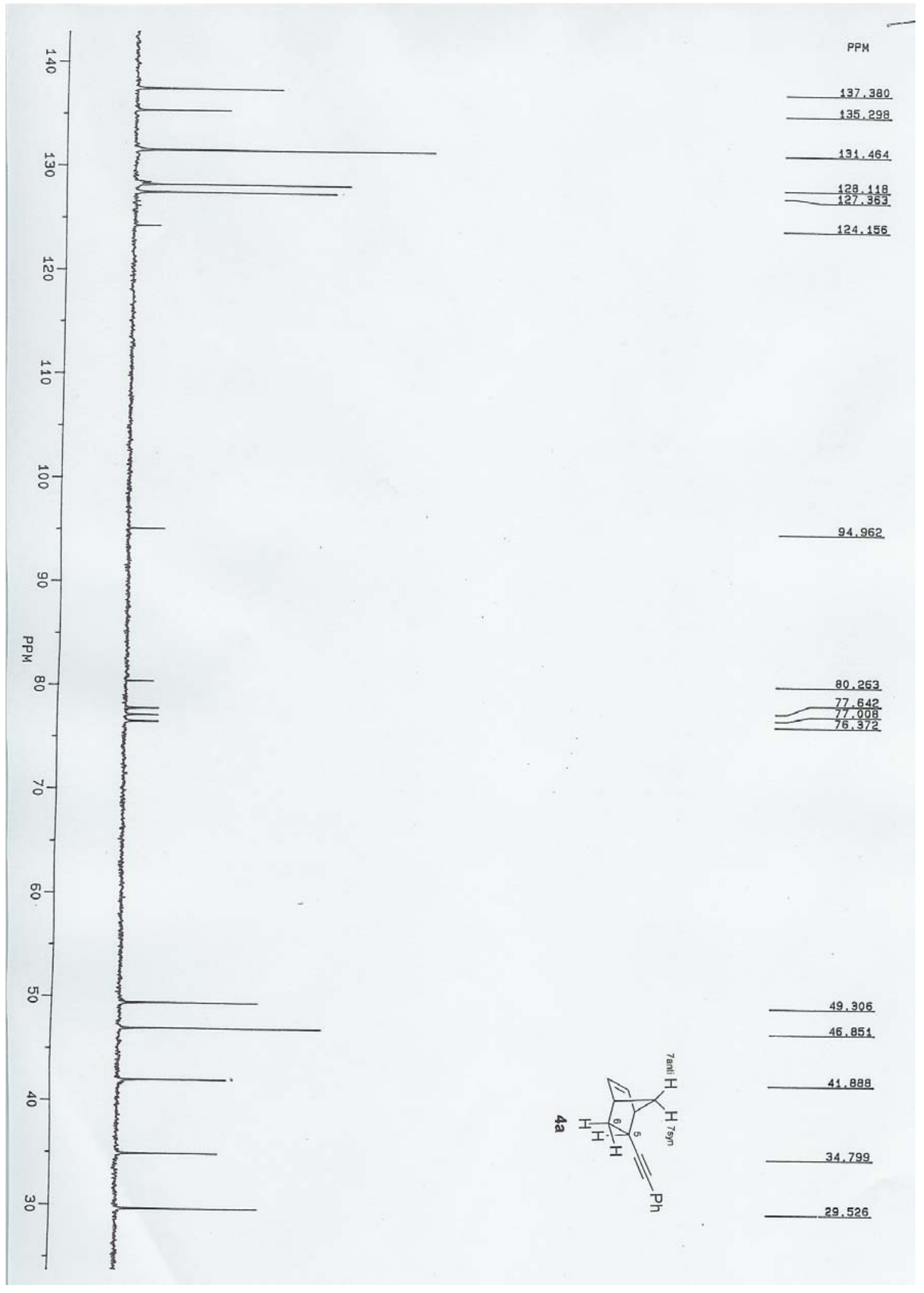




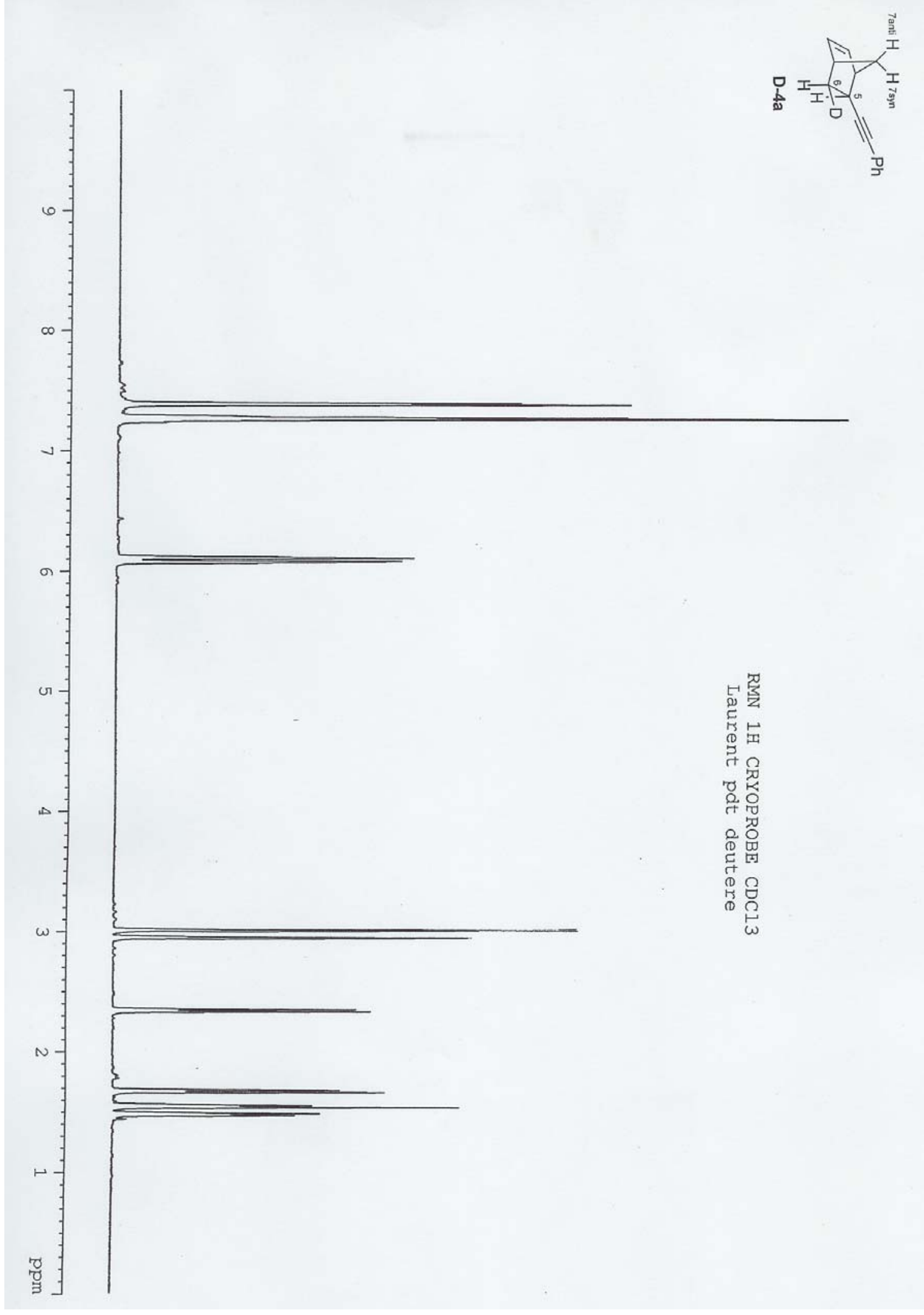




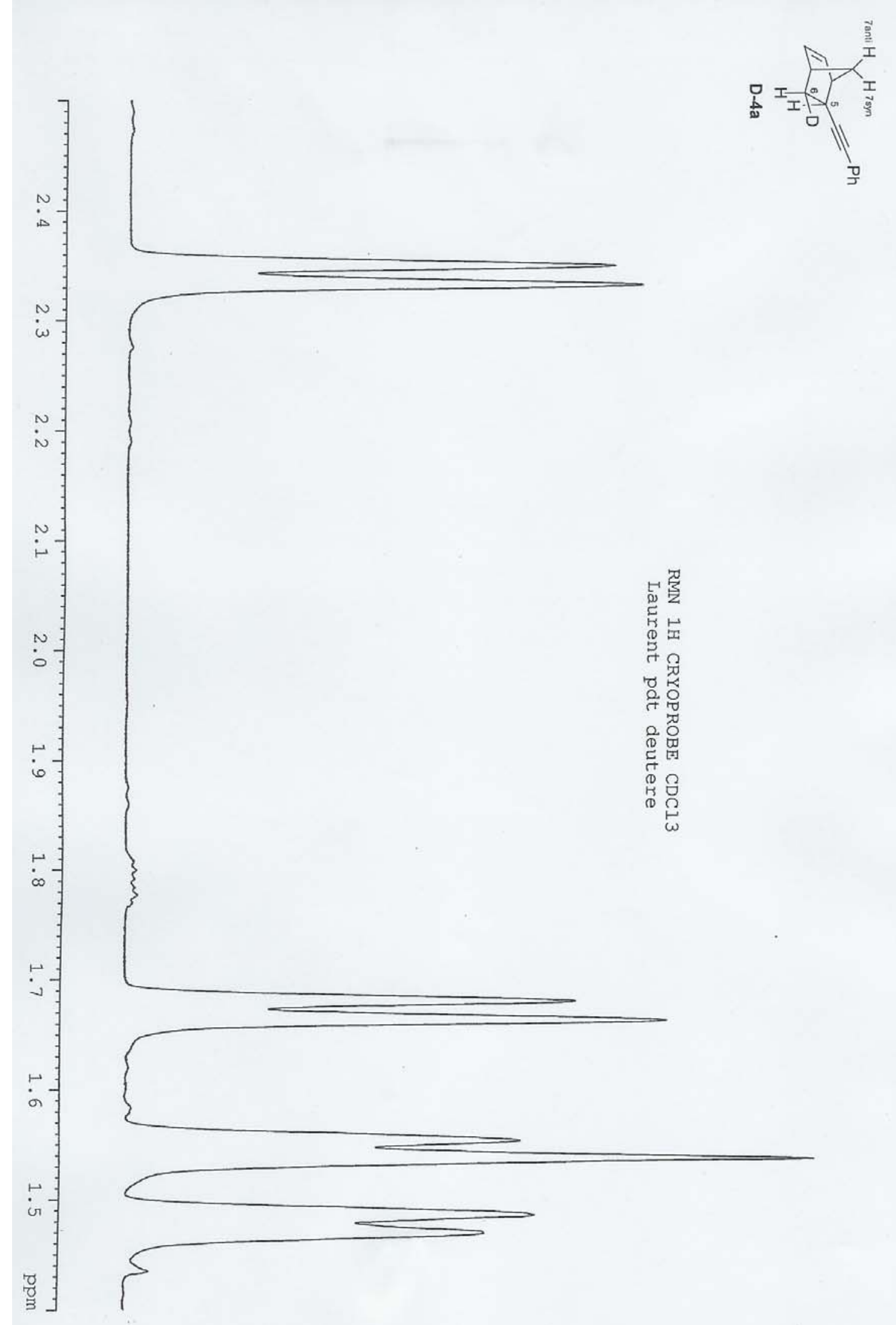




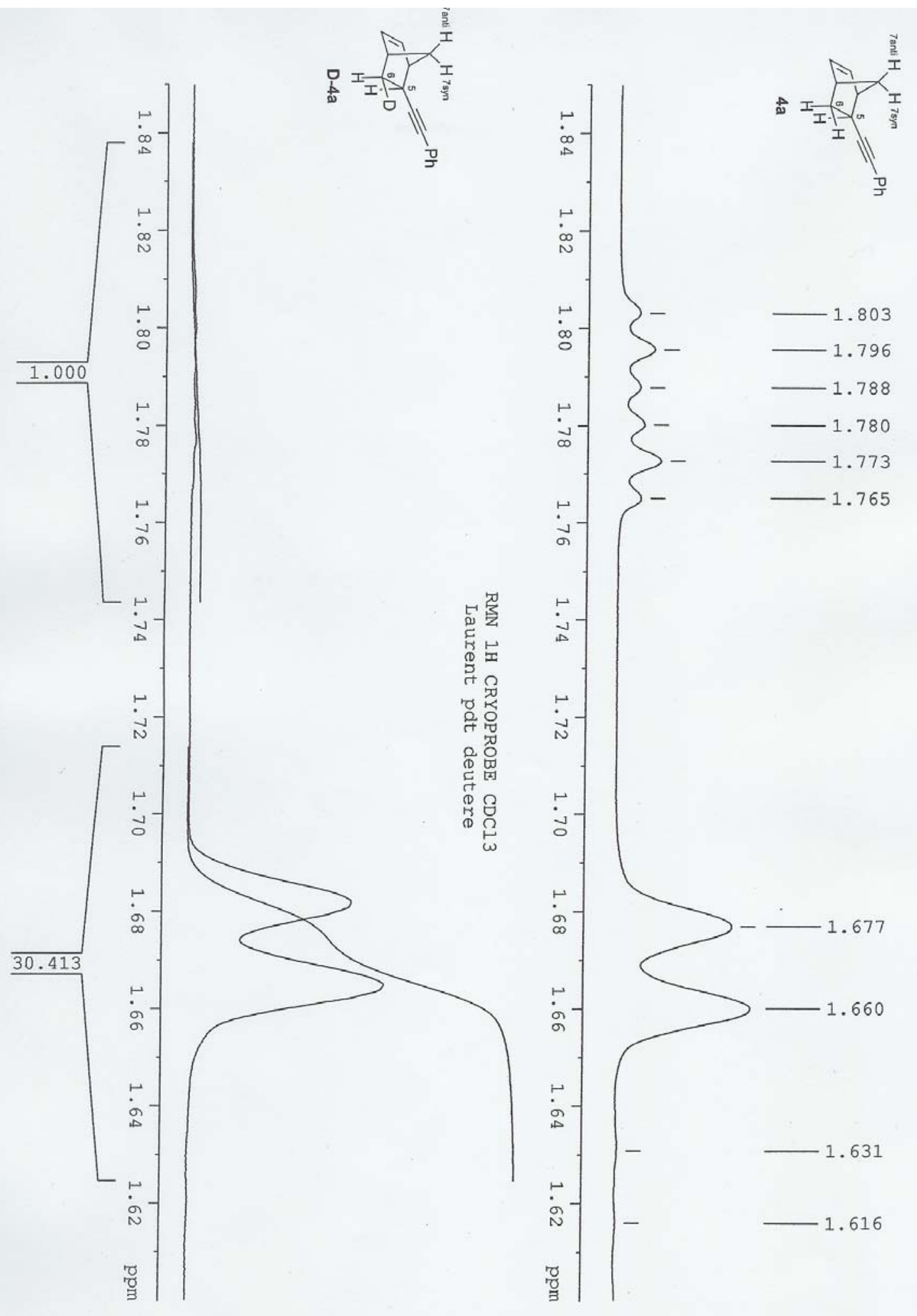




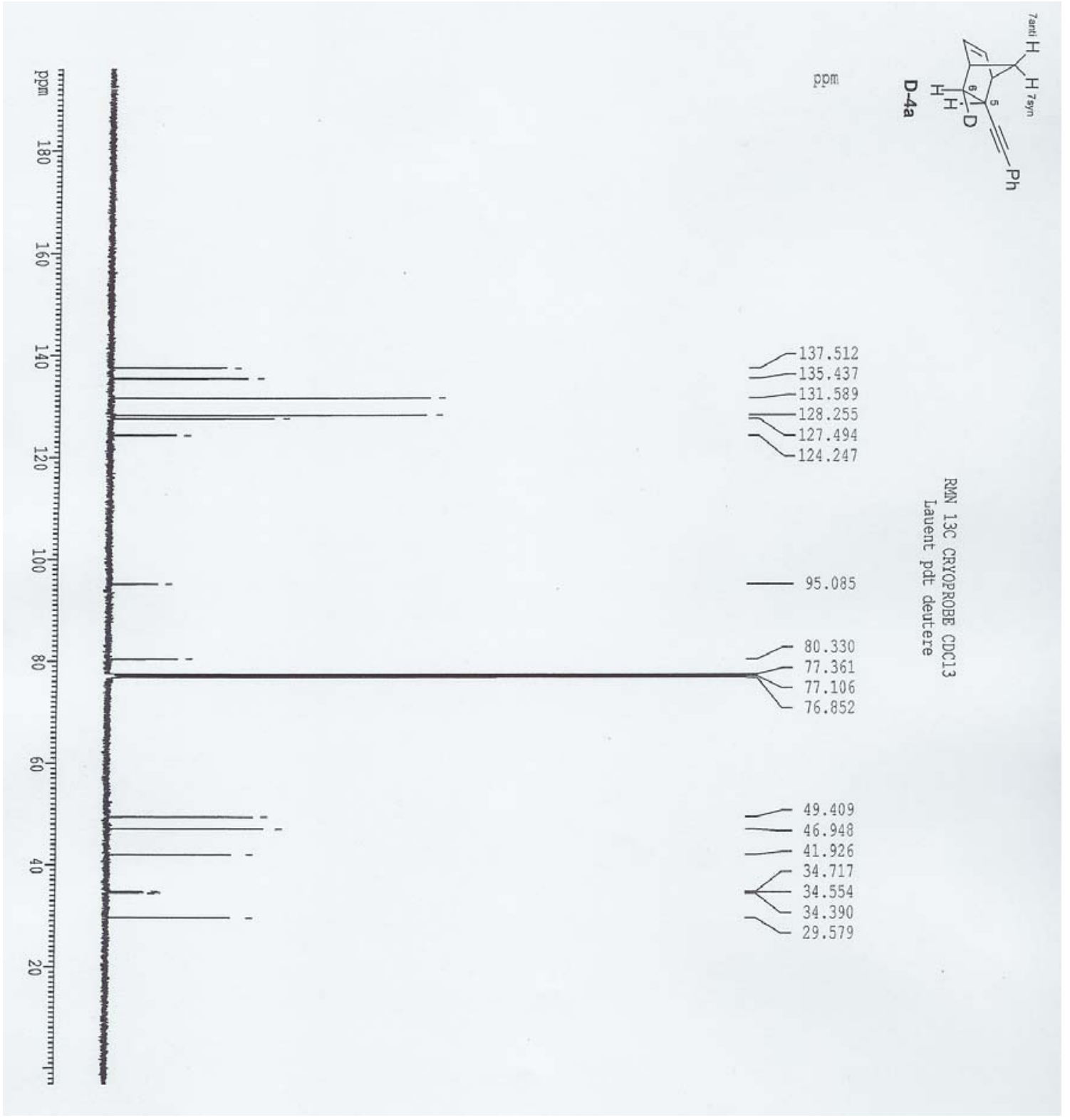




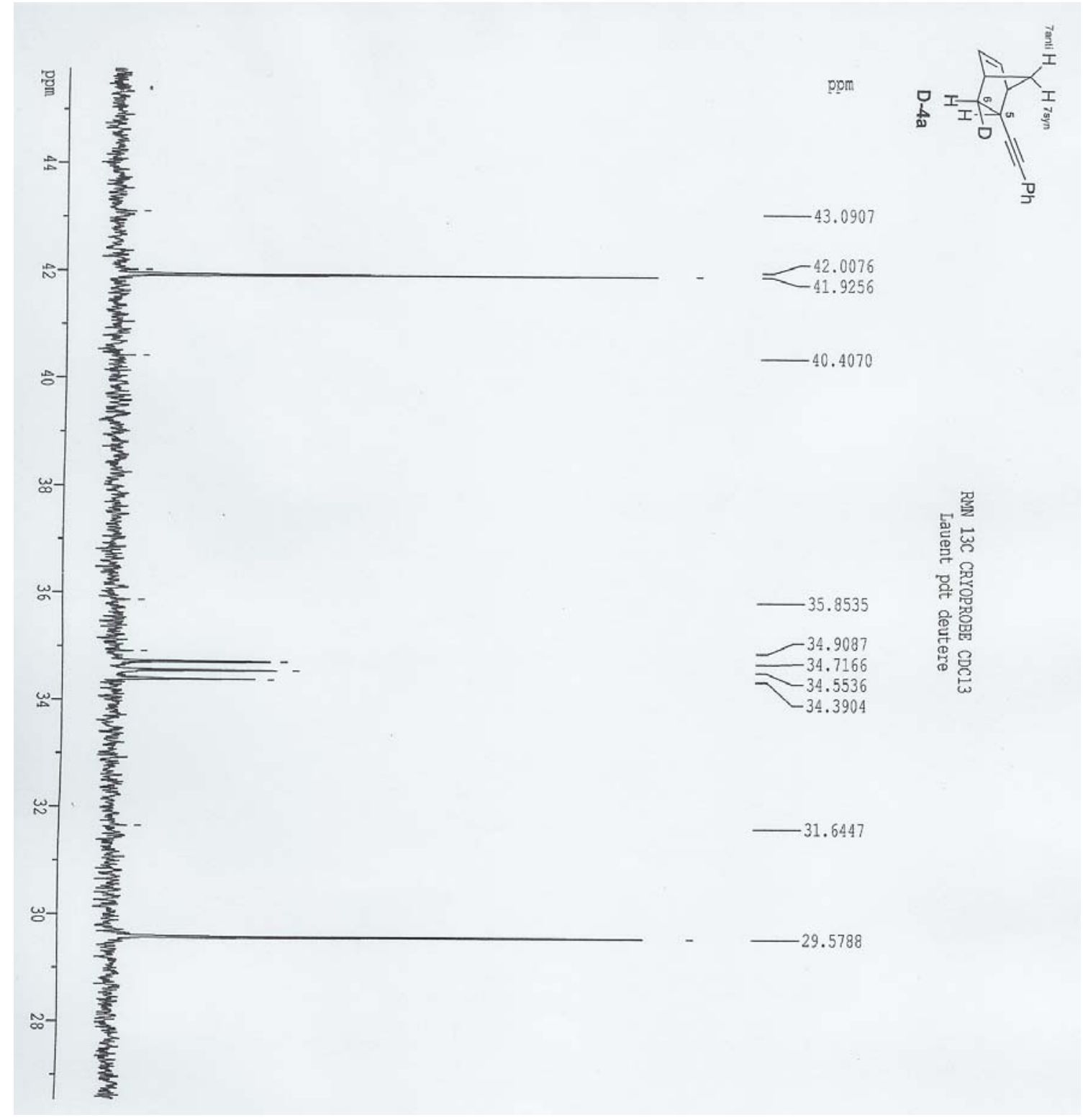

